\title{
Monitoring of the cometary activity of distant comet C/2006 S3 (LONEOS)^
}

\author{
P. Rousselot ${ }^{1}$, P. P. Korsun ${ }^{2}$, I. V. Kulyk ${ }^{2}$, V. L. Afanasiev ${ }^{3}$, O. V. Ivanova ${ }^{2}$, A. V. Sergeev ${ }^{4,5}$, and S. F. Velichko ${ }^{4,5}$ \\ ${ }^{1}$ University of Franche-Comté, Observatoire des Sciences de l'Univers THETA, Institut UTINAM - UMR CNRS 6213, BP 1615, \\ 25010 Besançon Cedex, France \\ e-mail: rousselot@obs-besancon.fr \\ 2 Main Astronomical Observatory of NAS of Ukraine, 27 Akademika Zabolotnoho Street, 03680 Kyiv, Ukraine \\ 3 Special Astrophysical Observatory of the Russian AS, 369167 Nizhnij Arkhyz, Russia \\ ${ }^{4}$ Center AMER Observatory of National Academy of Sciences of Ukraine, 27 Akademica Zabolotnoho Street, 03680 Kyiv, Ukraine \\ 5 IC AMER Terskol Observatory, Terskol, 361605 Kabardino-Balkaria Republic, Russia
}

Received 16 May 2014 / Accepted 28 July 2014

\section{ABSTRACT}

\begin{abstract}
Context. The study of small bodies that have a cometary activity at a large heliocentric distance (larger than 5 au) is important for a better understanding of the physical properties of comets and their origin. Such studies require long-term monitoring and a significant observational effort.

Aims. Our goal is to monitor the cometary activity and search for possible emission lines of C/2006 S3 (LONEOS), which is a distant comet that was discovered in 2006 and passed perihelion at 5.13 au in April 2012.

Methods. We performed different observing runs with telescopes, ranging from $2 \mathrm{~m}$ to $6 \mathrm{~m}$ both in imaging and spectroscopic modes in the optical range, between 2006 and 2014.

Results. The comet C/2006 S3 (LONEOS) was a very active object with $A f \rho \sim 4000 \mathrm{~cm}$ at its maximum of activity, corresponding to a dust production rate of $82 \mathrm{~kg} \mathrm{~s}^{-1}$, considering a mean geometric albedo of 0.1 and grain outflow velocities in the range $2-22 \mathrm{~m} \mathrm{~s}$. It appeared to be more active after its perihelion than before and we did not manage to detect any emission lines. Upper limits for the main cometary species that have emission bands in the optical range are provided. Our dust environment modeling is in agreement with a collimated source of dust that appeared in 2013.
\end{abstract}

Key words. comets: general - comets: individual: C/2006 S3 (LONEOS)

\section{Introduction}

The physical nature of comets is known mainly from the investigations of bright comets observed close to the Earth and the Sun ( 1-2 au). Observations at large heliocentric distances (more than $5 \mathrm{au}$ ) are scarce and much more difficult. These observations are, nevertheless, very useful to obtain more information about the origin of comets as well as their relation with similar objects, such as Kuiper belt objects and Centaurs.

The physical mechanism that drives cometary activity is quite different at these large heliocentric distances. The usual cometary activity created by sublimation of water ice is excluded because this process can only be efficient at a few au from the Sun, inside Jupiter's orbit (Meech \& Svoren 2004). The main mechanisms that have been proposed to explain cometary activity of comets at large heliocentric distances are: the transition phase between amorphous and crystalline water ice (Prialnik 1992), the annealing of amorphous water ice (Meech et al. 2009), and the sublimation of more volatile admixtures like CO and/or $\mathrm{CO}_{2}$.

In this context, the study of the cometary activity of distant comets pre-perihelion, which have never been close enough to the Sun to experience significant heating, is very important to

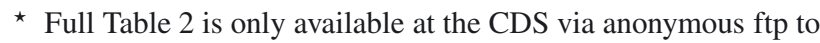
cdsarc.u-strasbg.fr (130.79.128.5) or via http://cdsarc.u-strasbg.fr/viz-bin/qcat?]/A+A/571/A73 place more observational constraints on the physical mechanism that drives cometary activity. So far, the number of such studies is very limited. Only a few comets have been studied inbound at heliocentric distances larger than 5 au (Korsun \& Chörny 2003; Tozzi et al. 2003; Meech et al. 2009; Korsun et al. 2010; Mazzotta Epifani et al. 2014; Shi et al. 2014). We present here an observational dataset of a distant comet observed in a large range of heliocentric distances.

Comet C/2006 S3 (LONEOS, hereafter S3) was discovered by the Lowell Observatory Near-Earth Object Search (LONEOS) team on 19 September 2006 at a heliocentric distance of 14.3 au (Skiff et al. 2006). At that time, the coma was estimated at 11 arcsec in diameter with a total $V$ magnitude of 19. Further orbital calculations provided a perihelion distance of $5.13 \mathrm{au}$, a perihelion passage on 16 April 2012, an orbital inclination of $166^{\circ}$, and a parabolic orbit. From the original orbital elements computed by Syuichi Nakano ${ }^{1}$ it is possible to classify S3 as a dynamically new comet, according to the classification proposed by Levison (1996), because $1 / a<10^{-4}$ au. Recently, Shi et al. (2014) presented a few photometric observational data on this target, obtained pre-perihelion at 5.86 au.

We performed a monitoring of the cometary activity of this distant comet between December 2006 and March 2014. We obtained both spectra in the optical range and photometric data with broadband filters. The comet S3 appears to be a very active

\footnotetext{
See www. oaa.gr.jp/ oaacs/nk.htm
} 
Table 1. Equipment of the observations.

\begin{tabular}{|c|c|c|c|c|c|c|c|c|}
\hline Telescope & $\begin{array}{l}\text { Diameter } \\
(\mathrm{m})\end{array}$ & $\mathrm{CCD}$ & $\begin{array}{l}\text { Pixel size } \\
(\mu \mathrm{m} \times \mu \mathrm{m})\end{array}$ & $\begin{array}{l}\text { Scale } \\
(" / \text { pix })\end{array}$ & $\begin{array}{l}\text { Field of view } \\
\left({ }^{\prime} \times{ }^{\prime}\right)\end{array}$ & Filter & $\begin{array}{c}\lambda_{\mathrm{c}} \\
\mathrm{nm}\end{array}$ & $\begin{array}{c}F W H M \\
\mathrm{~nm}\end{array}$ \\
\hline BTA & 6.0 & $2048 \times 2048$ & $13.5 \times 13.5$ & 0.18 (binned $2 \times 2)$ & $6.1 \times 6.1$ & $B, V, R c$ & $440,547,662$ & $97,79,150$ \\
\hline \multirow[t]{2}{*}{$\mathrm{RC}$} & 2.0 & $562 \times 512$ & $24 \times 24$ & 0.99 & $9.2 \times 8.4$ & $B, V, R X$ & $431,547,680$ & $100,90,111$ \\
\hline & & $2084 \times 2084$ & $24 \times 24$ & $0.31($ binned $3 \times 3)$ & $10.8 \times 10.8$ & $B, V, R X$ & $431,547,680$ & $100,90,111$ \\
\hline NOT & 2.5 & $2048 \times 2048$ & $13.5 \times 13.5$ & 0.19 & $6.5 \times 6.5$ & $B, V, R$ & $440,530,650$ & $100,80,130$ \\
\hline LT & 2.0 & $4096 \times 4112$ & $15 \times 15$ & $0.15($ binned $2 \times 2)$ & $10 \times 10$ & $B, V, R$ & $435,537,617$ & $100,90,130$ \\
\hline
\end{tabular}

Notes. BTA: 6 m Big Telescope Alt-azimuth (operated by the SAO). RC: 2 m Ritchey-Chretien Telescope (Peak Terskol). NOT: $2.5 \mathrm{~m}$ Nordic Optical Telescope (La Palma). LT: 2 m Liverpool Telescope (La Palma).

Table 2. Log of the observations.

\begin{tabular}{lccccllll}
\hline \hline Date of obs. & Exp. time $(\mathrm{s})$ & $\Delta(\mathrm{au})$ & $r(\mathrm{au})$ & Airmass & $\begin{array}{l}\text { Phase } \\
\text { angle }\left(^{\circ}\right)\end{array}$ & Data type & $\begin{array}{l}\text { Filter } / \\
\text { spectral range }(\AA)\end{array}$ & $\begin{array}{l}\text { Telescope } \\
\end{array}$ \\
\hline Dec. 13, 2006 & $2 \times 60 / 3 \times 180 / 2 \times 120$ & 13.67 & 13.85 & $1.25-1.26$ & 4.03 & image & $V / B / R \mathrm{c}$ & BTA \\
Dec. 13, 2006 & $4 \times 900$ & 13.67 & 13.85 & $1.26-1.35$ & 4.03 & spectrum & $3800-6800$ & BTA \\
Oct. 23, 2008 & $6 \times 120 / 8 \times 120 / 4 \times 120$ & 9.54 & 10.25 & $1.40-1.70$ & 4.05 & image & $V / R X / B$ & RC \\
Dec. 03, 2008 & 30 & 9.97 & 10.03 & 1.31 & 5.64 & image & $V$ & BTA \\
Dec. 03, 2008 & $3 \times 900$ & 9.97 & 10.03 & $1.32-1.36$ & 5.64 & spectrum & $3800-5400$ & BTA \\
Jun. 08, 2010 & $3 \times 60 / 3 \times 60 / 3 \times 60$ & 6.80 & 7.15 & $1.63-1.51$ & 7.82 & image & $V / R X / B$ & RC \\
Jul. 06, 2011 & $8 \times 60$ & 4.59 & 5.56 & $1.21-1.22$ & 3.25 & image & $R$ & NOT \\
Jul. 08, 2011 & $29 \times 60 / 4 \times 60 / 4 \times 60$ & 4.58 & 5.56 & $1.22-1.28$ & 3.08 & image & $R / V / B$ & NOT \\
Jul. 05, 2013 & $3 \times 60 / 60 / 60$ & 5.90 & 6.12 & $1.68-1.73$ & 9.47 & image & $R / B / V$ & NOT \\
Jul. 06, 2013 & $3 \times 60 / 60 / 60$ & 5.92 & 6.12 & $1.63-1.67$ & 9.49 & image & $R / B / V$ & NOT \\
Mar. 02, 2014 & $18 \times 20+16 / 9 \times 20 / 9 \times 20$ & 6.38 & 7.19 & $1.36-1.35$ & 4.91 & image & $R / V / B$ & LT \\
\hline
\end{tabular}

Notes. See Table 1 caption for telescope acronyms. The full Table is available at the CDS.

object, despite its large heliocentric distance, with Af $\rho$ increasing up to $\sim 4000 \mathrm{~cm}$ post-perihelion. In this paper, we present our observational data, the data reduction, and the analysis of the resulting data of this interesting target.

\section{Observational data}

The monitoring of S3 has been carried out with different telescopes. We obtained spectra with the $6 \mathrm{~m}$ Big Telescope Alt-azimuth (BTA) located in the Caucasus and operated by the Special Astrophysical Observatory (SAO) of the Russian Academy of Science (Russia). We obtained photometric data with the $6 \mathrm{~m}$ BTA, the $2 \mathrm{~m}$ Ritchey-Chretien Telescope located at Peak Terskol (International Center for Astronomical and Medico-Ecological Research, Ukraine, Russia), the $2.5 \mathrm{~m}$ Nordic Optical Telescope (NOT) located at La Palma (Spain), and the $2 \mathrm{~m}$ robotic Liverpool Telescope also located at La Palma. The main technical parameters of the telescopes and CCD detectors are presented in Table 1 and the observing log is presented in Table 2.

$6 \mathrm{~m}$ BTA, SAO. Spectroscopic and photometric observations of S3 were made on December 13, 2006 and repeated on December 3, 2008 with the multimode focal reducer SCORPIO attached to the prime focus of the telescope. It operated in both photometric mode and long-slit spectroscopic mode (Afanasiev $\&$ Moiseev 2005). To increase the signal/noise ratio of the measured signal, we applied on-chip binning of 2 in spatial directions to the photometric and spectroscopic frames. We obtained spectra with a grism using volume phase holographic grating (VPHG) combined with a long, narrow-slit mask. We used the VPHG550G grism and the slit with dimensions of 1 .'0 $\times 6$ ' 1 in 2006 . These provide an effective wavelength region of 3800-6800 $\AA$ with $10 \AA$ of spectral resolution. In 2008, the VPHG1200B grism with the $11^{\prime \prime} 0 \times 6$ '. 1 slit provided a spectral range of 3800-5400 $\AA$ with a spectral resolution of $5 \AA$. The telescope was tracked on the comet to compensate its apparent movement during the exposures. We observed the spectrophotometric standard star BD+25d4655 to fulfill absolute calibration (Oke 1990).

We observed twilight morning sky to provide flatfield corrections of the images, while a smoothed spectrum of an incandescent lamp was observed for the spectral data. We obtained spectra of an $\mathrm{He}-\mathrm{Ne}-\mathrm{Ar}$ lamp to perform wavelength calibrations.

On December 13, 2006 we obtained the photometric data through the $B, V$, and $R$ broadband filters. We observed a field of standard stars (PG 0231) at approximately the same airmasses as the comet airmasses to perform the photometric calibration (Landolt 1992). A reference zero point and color term for transformation to the standard photometric system were calculated. The uncertainty of the regression line was better than 0.02 mag on average. The difference in the first order member of extinction between the comet and the standard stars was considered small and compensated for by the mean monochromatic extinction coefficients measured for Peak Terskol Observatory, which is located in the same mountain area at an altitude of $3100 \mathrm{~m}$ (Kulyk et al. 2004). During the observing run conducted in 2008, we obtained the images through the $V$ filter only. We observed the standard star BD+25d4655 to carry out the absolute photometric calibration. The difference in airmasses between the comet and standard star was compensated with the mean extinction coefficient. The color member of the extinction calculated for $V$-band was neglected because it is small and comparable to the uncertainty of the main extinction coefficient itself. 
2 m RC, Peak Terskol. We carried out two observing runs at Peak Terskol. During the 2008 observing period we used the focal reducer attached to the $2 \mathrm{~m} \mathrm{RC}$ Telescope. We observed the spectrophotometric standard stars HD 15318, HD 30739, HD 224926, HD 188350 in a wide airmass range (Bessell 1999). The stars were processed together to calculate a zero point for the instrumental magnitudes and mean coefficients of atmospheric extinction for all the filters. A root mean square scatter around the regression line was $0.025 \mathrm{mag}$ in average. The telescope was guided on a non sidereal rate to compensate the comet motion during the exposures. We obtained a set of exposures from the evening and morning twilight sky through all the filters to create flatfield images.

In 2010, the observing period comprised several photometric nights, however, the comet was only successfully observed on June 08/09. We processed the images of the standard star BD+33d2642 obtained during the different nights together to control the mean extinction coefficients and reference zero points for three color bands. The root mean square errors were approximately equal to $0.03 \mathrm{mag}$ on average. The usual set of flatfield images was obtained.

$2.5 \mathrm{~m}$ NOT, La Palma. The photometric observations were made just before and after the perihelion passage, which occurred on 23 April 2012. Images were obtained with the Andalucia Faint Object Spectrograph and Camera (ALFOSC) used in imaging mode. In this mode, the field of view is $6.4 \times 6.4$ using a $2048 \times 2048$ CCD detector with a $13.5 \mu$ m pixel size, corresponding to $0{ }^{\prime} 19$ on the sky. We conducted the observations with broadband Bessell $B V R$ filters and we also observed a field with standard stars (SA 110) at different airmasses to provide information for the photometric reduction (Landolt 1992). We obtained the observations of July 6, 2011 in a crowded field with poor seeing $\left(\sim 2^{\prime \prime}\right)$, while the observing conditions were much better on July 8, 2011 (seeing 0.' 8) and July 05/06, 2011 (seeing also better than $1^{\prime \prime}$ and photometric conditions).

$2 \mathrm{~m}$ LT, La Palma. The photometric observations were made with the $2 \mathrm{~m}$ robotic Liverpool telescope with broadband Bessell $B$, Bessell $V$, and SDSS $R$ filters. Because of poor seeing $\left(\sim 2^{\prime \prime}\right)$, the images were binned $2 \times 2$. A field of standard stars (PG1323-086) was also observed at a similar airmass to provide zero point photometric coefficients.

\section{Data reduction}

\subsection{Spectra reduction}

The observational data were processed using the package Scorpio2k.lib developed at SAO to reduce long-slit spectra observed with SCORPIO. An averaged bias frame was removed from the data. We compensated a nonuniform sensitivity of the chip's pixels for using expositions of the lamp with smoothly varying energy distribution. We made also geometric correction of the raw frames. Wavelength calibration was done by using exposures of a $\mathrm{He}-\mathrm{Ne}-\mathrm{Ar}$ lamp.

We removed the night sky spectrum existing in the frames with the cometary spectra by measuring its level in each column over the zones free of the cometary coma. We made a photometric calibration of the cometary spectra using observed spectra of the spectrophotometric standard star. Spectral behavior of atmospheric extinction was taken from Kartasheva \& Chunakova (1978) and Kulyk et al. (2004).

The cosmic ray events were removed by applying median filtering between the frames for each pixel values. This processing reduced the noise and improved the final signal-to-noise ratio on the spectra.

\subsection{Photometric data}

For all the photometric data standard data processing was done to preprocess the data: we subtracted bias images from the scientific images and divided the resulting frames with flatfield images. In the case of the Liverpool Telescope we used the preprocessed data provided by the reduction pipeline of this telescope.

The second step consisted in computing all the photometric coefficients, thanks to the standard star images, as explained in the previous section for the Peak Terskol and SAO data. In the case of the NOT data, we used the IRAF package to compute all the photometric coefficients. For the Liverpool Telescope data, only zero point could be computed, because standard star images could only be obtained at one airmass. For the extinction coefficients, we used an average of the one obtained for the NOT telescope, located in the same obervatory. Because of the very small difference in airmass with the scientific exposures (less than 0.1 airmass), the uncertainty of the extinction coefficient had very little influence.

For all the photometric data, we computed averaged images for each observing night: one for that target and one for a reference star chosen to have a good signal/noise ratio and no other star too close to it. In both cases, we shifted the images in such a way that the target or the star remains exactly in the selected sub-frame (with a $401 \times 401$ pixels). For the images obtained on July 6, 2011, median images efficiently removed background stars.

From the averaged subframes obtained over all nights, we computed radial profiles calibrated in units of magnitude/arsec ${ }^{2}$ both for the target and the reference star, thanks to the photometric coefficients. The comparison of the profiles for the target and the star permitted us to confirm the importance of the coma.

\section{Analysis}

\subsection{Spectra}

The resulting spectra were coadded in the spatial direction when a coma was detected. As the coma was sufficiently condensed during the observation, integration in the spatial direction was limited to $20^{\prime \prime}$. The continuum components of the onedimensional cometary spectra show a reddening effect. To fit the continuum we used a solar spectrum, which was taken from Neckel \& Labs (1984). It was degraded to the resolution of our observations by convolving the solar spectrum with the appropriate instrumental profile and normalized to the flux from the comet around $5000 \AA$. The proper spectral behavior was gained multiplying the solar spectrum by a polynomial. Derived continua, which are superimposed on the spectra observed on December 13, 2006 and December 3, 2008, are depicted in Figs. 2A and 3A, respectively. The polynomials used are displayed in Figs. 2C and 3C.

Subtracting the calculated continuum from the observed spectrum, we obtained a residual signal, in which emission features would be detected. These residuals can be seen in Figs. 2B and $3 \mathrm{~B}$, where $\pm 3 \sigma$ levels are also plotted. Their inspection shows that no regular features exceed the $3 \sigma$ level. Therefore, we concluded that we have not detected emissions in the spectra. No emissions were also detected in a low resolution spectrum of S3 obtained at Castanet-Tolosan Observatory (France) 


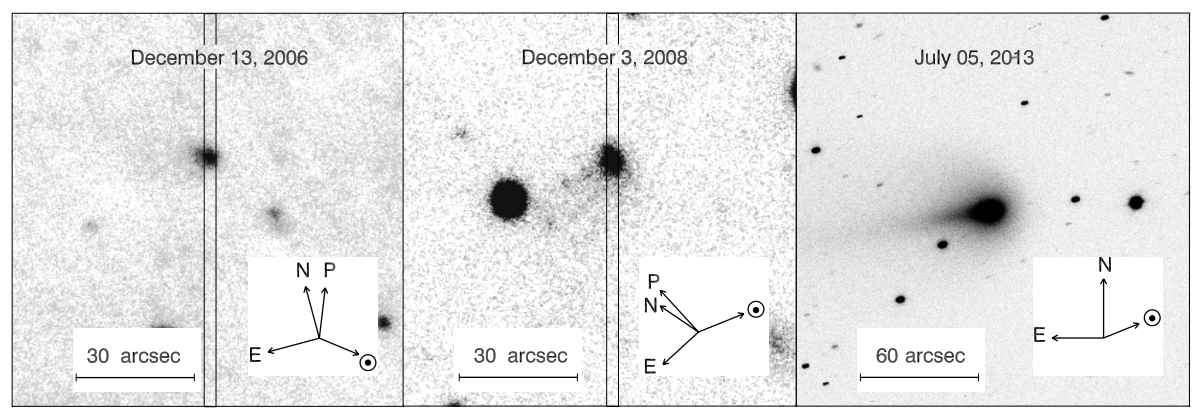

Fig. 1. Appearance of S3 within the monitoring. North $(\mathrm{N})$, east $(\mathrm{E})$, differential atmospheric refraction direction $(\mathrm{P})$, sunward direction $(\odot)$, and scale bar are indicated. The vertical narrow box across the comet marks the position of the slit in the spectroscopic mode.

Table 3. Upper limits for the main cometary species not detected in the spectra.

\begin{tabular}{lcccc}
\hline \hline Molecule & $\begin{array}{c}\text { Central wavelength, } \\
\AA / \Delta \lambda\end{array}$ & $\begin{array}{c}\sigma, \\
10^{-17} \mathrm{erg} \mathrm{s}^{-1} \mathrm{~cm}^{-2} \AA^{-1}\end{array}$ & $\begin{array}{c}\text { Flux, } \\
10^{-17} \mathrm{erg} \mathrm{s}^{-1} \mathrm{~cm}^{-2}\end{array}$ & $\begin{array}{c}\text { Gas production rate, } \\
10^{23} \mathrm{~mol} \mathrm{~s}^{-1}\end{array}$ \\
\hline 2006 & $3870 / 62$ & 0.46 & & \\
$\mathrm{CN}$ & $4062 / 62$ & 0.22 & $<5.0$ & $<4.3$ \\
$\mathrm{C}_{3}$ & $4266 / 64$ & 0.15 & $<2.4$ & $<0.3$ \\
$\mathrm{CO}^{+}$ & $5141 / 118$ & 0.11 & $<1.6$ & $<6.2$ \\
$\mathrm{C}_{2}$ & $3870 / 62$ & 0.56 & $<1.2$ & $<2.0$ \\
\hline 2008 & $4062 / 62$ & 0.38 & $<3.0$ & $<0.1$ \\
$\mathrm{CN}$ & $4266 / 64$ & 0.38 & $<2.1$ & $<3.3$ \\
$\mathrm{C}_{3}$ & $5141 / 118$ & 0.28 & $<2.1$ & \\
$\mathrm{CO}^{+}$ & & & $<1.5$ & \\
$\mathrm{C}_{2}$ & & &
\end{tabular}

Notes. These values have been computed with an aperture of $1 \times 20^{\prime \prime}$.

when it was at a heliocentric distance of 5.8 au after perihelion passage ${ }^{2}$.

We determined upper limits to the emission fluxes and upper limits to the production rates of the neutrals. To obtain the upper limits to the emission fluxes, we adopted a Gaussian having a FWHM equal to the spectral resolution as an equivalent of the minimal measurable signal. The amplitude of the Gaussian was equal to the RMS noise level calculated within wavelength regions associated with the bandpass of the narrowband cometary filters (Farnham et al. 2000). The upper limits to the production rates were derived using a Haser model (Haser 1957). The model parameters were taken from Langland-Shula \& Smith (2011). The upper limits to the fluxes and gas production rates are listed in Table 3.

The derived polynomials can be used to measure the reddening effect if the slit is oriented along the parallactic angle. The slit was deviated by $15^{\circ}$ relative to the north in a clockwise direction and the mean parallactic angle was 21.7 when the spectra were observed in 2006 (see Fig. 1). Consequently, the expected elongation of the cometary coma due to differential atmospheric refraction was inclined by 6.7 relative to the slit direction. The amount of the elongation, about $1^{\prime \prime}$, was taken from Filippenko (1982). A fraction of the light is lost with a direction normal to the slit orientation. The projection of the elongation on this direction is 0 ' 1 , which corresponds to half of a pixel. Taking into account that the coma of the comet is an extended source, the slit has appreciable width, and the seeing was $2 . .5$, we expected that the differential atmospheric refraction would not have crucial influence on the real slope of the spectra. Figure 4 is an illustration of the conclusion. Here the surface profile of the cometary coma, which was taken from the image observed through the $V$ filter and calculated along the direction normal to the slit, and

2 http://www.astrosurf.com/buil/comet/C2006_S3/obs.htm

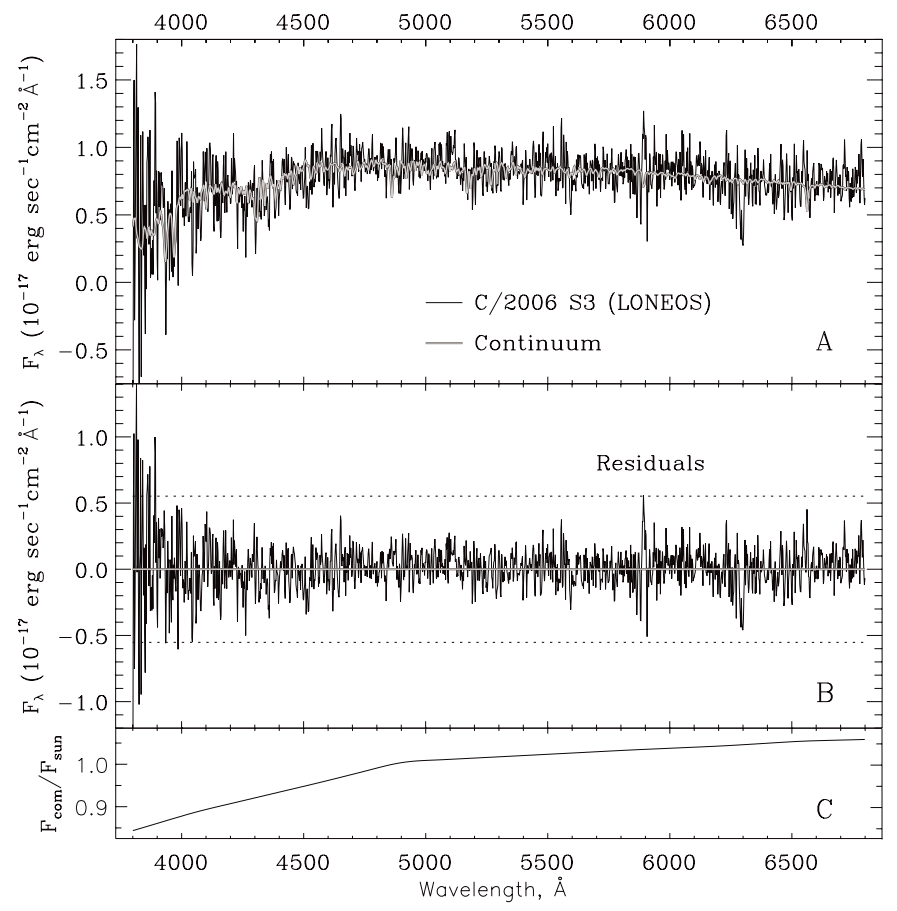

Fig. 2. Spectrum of $S 3$ observed at a heliocentric distance of 13.85 au: A) the observed spectrum with the superimposed continuum; B) the "observed spectrum - fitted continuum"; C) the polynomial correction of the solar spectrum.

the slit bounds are displayed. The signal does not significantly vary within the slit because of uncertainty in the slit position amounting to 0 ' $^{\prime} 1$. Consequently, we can use the polynomial to derive the reddening of the spectrum. The polynomial shows two branches with linear dependence on wavelengths, namely, 


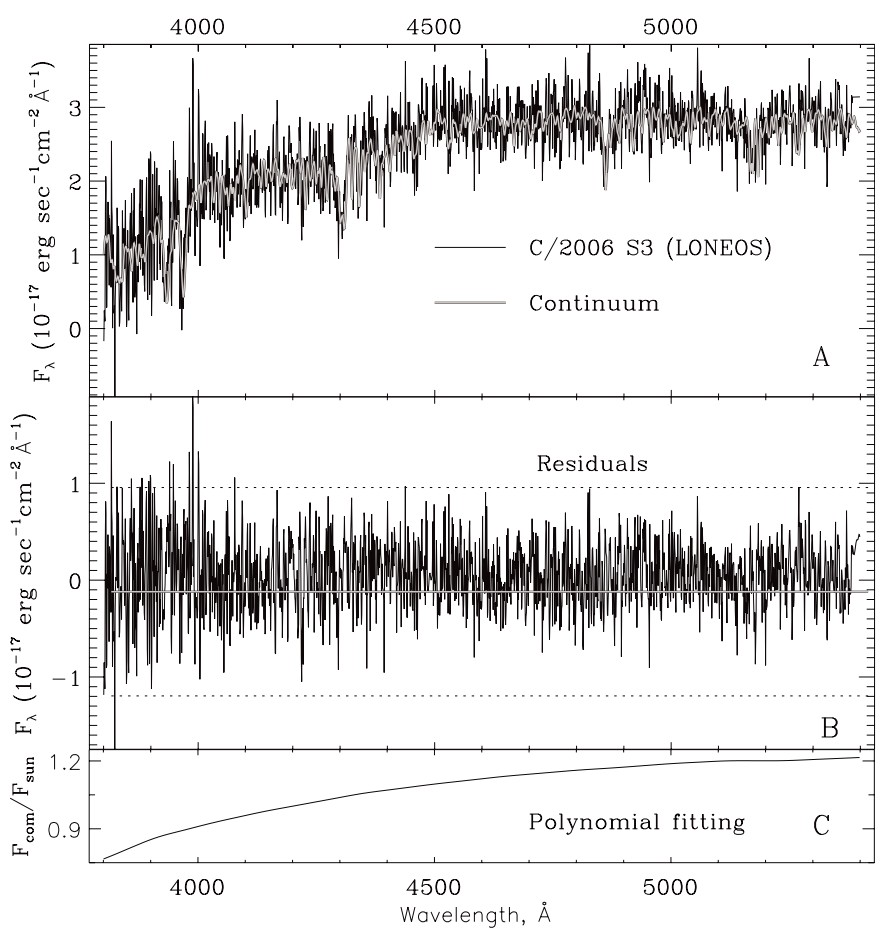

Fig. 3. Spectrum of S3 observed at a heliocentric distance of $10.03 \mathrm{au}$ : A) the observed spectrum with the superimposed continuum; B) the "observed spectrum - fitted continuum"; C) the polynomial correction of the solar spectrum.

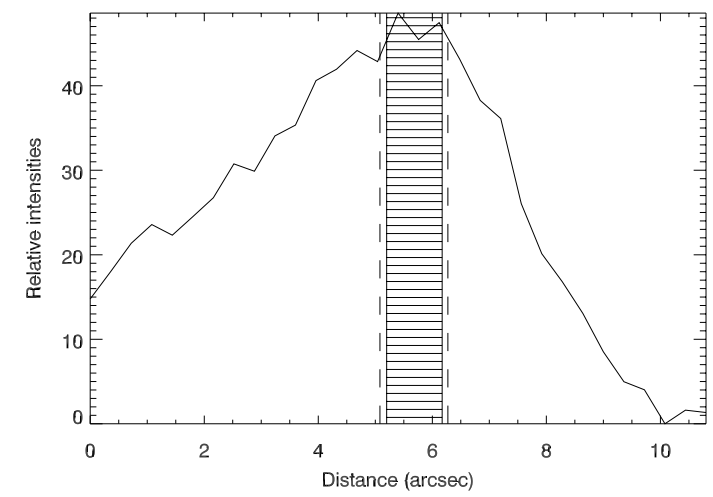

Fig. 4. Surface profile of the cometary coma measured along the direction normal to the slit. Vertical dashed strip marks the position of the slit, and vertical dashed lines indicate the uncertainty of the slit position due to the differential atmospheric refraction.

$15.1 \pm 4.9 \% / 1000 \AA$ within the 3800-4900 $\AA$ wavelength region and $2.8 \pm 0.3 \% / 1000 \AA$ within the 4900-6800 $\AA$ wavelength region.

For the data obtained on December 3, 2008, the elongation of the cometary coma due to differential atmospheric refraction was inclined relative to the slit direction by $34^{\circ} 2$ (see Fig. 1), and it was not possible to derive the reddening of the dust accurately for these data.

\subsection{Photometric data}

In all the images of $\mathrm{S} 3$, a coma was clearly visible around the nucleus. In order to measure the cometary activity accurately, we used the averaged images obtained each night to derive $A f \rho$ values. This parameter was introduced by A'Hearn et al. (1984) to measure cometary activity in such a way that it does not differ

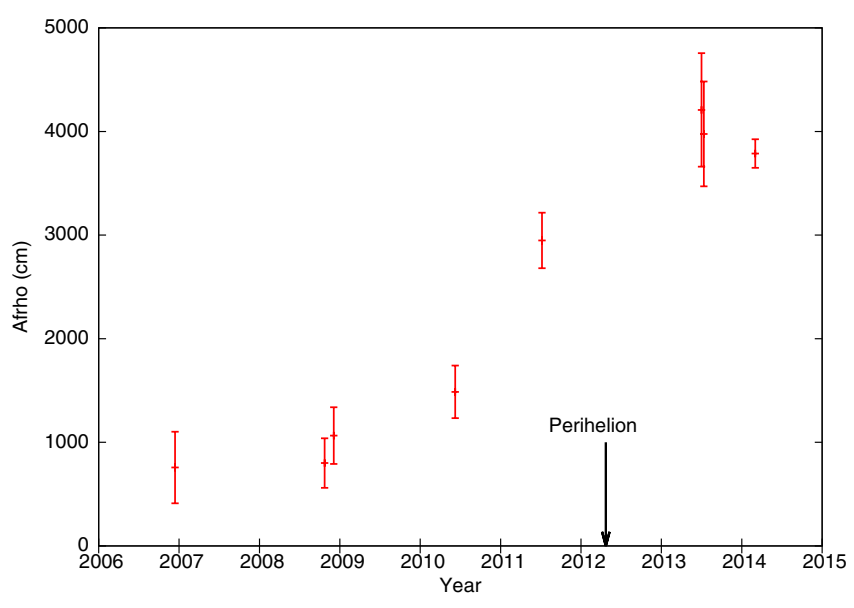

Fig. 5. Af $\rho$ values obtained for all the photometric data in the $V$ filter. They have been computed by averaging the values in the cometocentric range $5-20^{\prime \prime}$.

too much from one observer to another and to be independent of unknown parameters, such as grain albedo or grain size. It is a product of the albedo $(A)$, the filling factor $(f)$, i.e., the ratio of the total cross section of grains within the field of view by the area of the field of view and $\rho$, the linear radius of the field of view at the comet distance. For a given band, it is possible to compute it from the magnitude of the Sun in the same band. We can write

$A f \rho=\frac{2.4686 \times 10^{19} r^{2} \Delta 10^{0.4\left(M_{\mathrm{Sun}}-M\right)}}{D}$

where $r$ is the heliocentric distance (au), $\Delta$ the geocentric distance (au), $M_{\text {Sun }}$ the magnitude of the Sun in the considered filter, $M$ the overall magnitude of the coma, and $D$ the apparent diameter of the field of view expressed in arcsec. The resulting Af $\rho$ value is expressed in $\mathrm{cm}$ and is supposed to be, more or less, independent of the aperture size used to measure it (if the radial profile follows a $\rho^{-1}$ dependence).

We computed the Af $\rho$ parameter with radial distance for all the averaged images containing $\mathrm{S} 3$ thanks to the radial profiles computed with the photometric coefficients (see Sect. 3.2). Because the $A f \rho$ values change with cometocentric distance, we computed an average value in an interval varying from 5 to $20^{\prime \prime}$. This interval corresponds to values computed outside the seeing disk and with a relatively good signal/noise ratio for the coma. The error bars computed correspond to the range of Af $\rho$ values in this cometocentric interval. When these data are compared to the results published by Shi et al. (2014), obtained on April 2011, they appear to be in good agreement both for the color indices and the $A f \rho$ values.

Figure 5 presents an overall view of the results obtained with all our photometric data. Table 4 presents the detail of the Af $\rho$ values, total magnitudes, and color indices computed from our photometric data. Figure 6 presents the $A f \rho$ profiles obtained for the three filters during the 2013 NOT observing run, i.e., for our data with the higher level of cometary activity.

Figure 5 demonstrates that S3 was more active after its perihelion than before. It can also be seen that its level of activity was very important when compared to similar targets. For example, Mazzotta Epifani et al. (2014) present observational results on eight different long-period comets observed far from the Sun, i.e., at similar heliocentric distances (between 5 and 8.5 au, for these authors). All these targets have much lower Af $\rho$ values, 
Table 4. Af $\rho$ values (expressed in $\mathrm{cm}$ ), total magnitudes, and color indices computed from our photometric data.

\begin{tabular}{lcccccccc}
\hline \hline Date & $A f \rho B$ band & $A f \rho V$ band & $A f \rho R$ band & $B$ & $V$ & $R$ & $B-V$ & $V-R$ \\
\hline Dec. 13, 2006 & $651 \pm 273$ & $757 \pm 345$ & $737 \pm 401$ & $20.0 \pm 0.2$ & $19.5 \pm 0.2$ & $19.1 \pm 0.2$ & $0.79 \pm 0.04$ & $0.62 \pm 0.03$ \\
Oct. 23, 2008 & $1014 \pm 266$ & $800 \pm 239$ & $865 \pm 283$ & $18.4 \pm 0.2$ & $18.1 \pm 0.2$ & $17.8 \pm 0.2$ & $0.47 \pm 0.04$ & $0.58 \pm 0.03$ \\
Dec. 3, 2008 & & $1065 \pm 274$ & & & $17.8 \pm 0.2$ & & & \\
June 8, 2010 & $1553 \pm 184$ & $1487 \pm 254$ & $1620 \pm 194$ & $16.6 \pm 0.2$ & $16.2 \pm 0.2$ & $15.8 \pm 0.2$ & $0.68 \pm 0.05$ & $0.51 \pm 0.04$ \\
Jul. 6, 2011 & & & $2661 \pm 256$ & & & $13.8 \pm 0.3$ & & \\
Jul. 8, 2011 & $2638 \pm 322$ & $2948 \pm 268$ & $2767 \pm 228$ & $15.1 \pm 0.3$ & $14.3 \pm 0.3$ & $13.8 \pm 0.3$ & $0.74 \pm 0.06$ & $0.43 \pm 0.06$ \\
Jul. 5, 2013 & $4140 \pm 568$ & $4208 \pm 548$ & $3911 \pm 512$ & $15.0 \pm 0.3$ & $14.4 \pm 0.2$ & $14.0 \pm 0.2$ & $0.66 \pm 0.03$ & $0.44 \pm 0.02$ \\
Jul. 6, 2013 & $3918 \pm 476$ & $3976 \pm 506$ & $3858 \pm 483$ & $15.1 \pm 0.2$ & $14.4 \pm 0.2$ & $13.9 \pm 0.2$ & $0.67 \pm 0.03$ & $0.49 \pm 0.02$ \\
Mar. 2, 2014 & $3668 \pm 216$ & $3787 \pm 138$ & $3928 \pm 208$ & $15.6 \pm 0.2$ & $14.9 \pm 0.2$ & $14.3 \pm 0.2$ & $0.66 \pm 0.06$ & $0.57 \pm 0.06$ \\
\hline
\end{tabular}

Notes. The total magnitudes correspond to the magnitudes measured with a large radius $(\sim 30 \mathrm{arcsec})$ corresponding to a constant magnitude for larger apertures. The color indices are computed with a 5-arcsec aperture. See the text for more details.

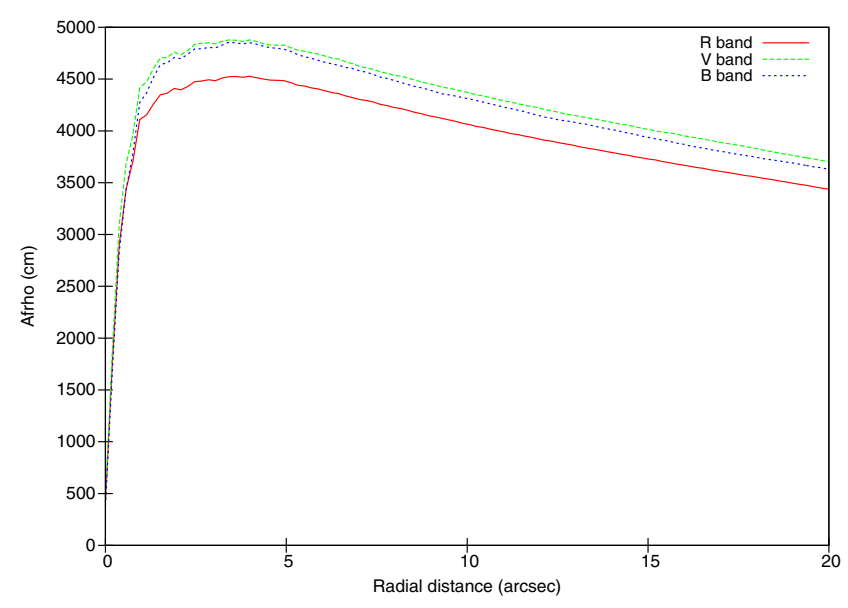

Fig. 6. Af $\rho$ values obtained with the NOT data of 5 July, 2013 as a function of the radial distance.

typically a few hundreds of cm except one (C/2005 L3) with Af $\rho \sim 5000 \mathrm{~cm}$ at 6.64 au. The cometary activity of S3 remains, nevertheless, well below the exceptional level of cometary activity of $174 \mathrm{P} /$ Echeclus during its main outburst in 2007. At that time, the $A f \rho$ value of $174 \mathrm{P}$ was about $7000 \mathrm{~cm}$ (Rousselot 2008) at 12.92 au from the Sun.

From the magnitudes of Table 4 , it is also possible to estimate how rapidly the cometary activity changes with heliocentric distance. The way the brightness of a comet varies is usually expressed by this formula (Marsden \& Roemer 1982):

$m_{1}=H_{1}+2.5 n \log (r)+2.5 k \log (\Delta)$

where $m_{1}$ is the apparent total magnitude, $H_{1}$ its absolute total magnitude (corresponding to a geocentric and heliocentric distances equal to $1 \mathrm{au}$ ), $r$ the heliocentric distance (au), and $\Delta$ the geocentric distance (au). The parameters $n$ and $k$ are a measure of the sensitivity of the magnitude variation to $r$ and $\Delta$. Generally, $k$ is taken equal to 2 but $n$ presents large variations according to the cometary activity and is often taken equal to 4 for the comets in the range of heliocentric distance in which they present an important cometary activity.

From the $V$-magnitudes of Table 4 , and using $k=2$, we can compute that $n \sim 2.5$ in the range $\sim 14-7$ au, $n \sim 3.8$ in the range $\sim 7-5.5$ au (pre-perihelion), and $n \sim 2$ in the range $\sim 5.5-6.1$ au (post-perihelion). These values show that S3 presented the largest sensitivity to the heliocentric distance in the range $\sim 7-5.5$ au (pre-perihelion). Just after perihelion it is not really sensitive to the variation of heliocentric distance, which was also the case when it was far from the Sun.

\subsection{Dust mass production rate}

In order to reduce the $A f \rho$ parameter to the dust mass loss rate, we used an approach described by Newburn et al. (1981), Newburn \& Spinrad (1985), and Weiler et al. (2003), taking into account the discussion by Fink \& Rubin (2012). The final equation to calculate the dust mass production rate is the following (Weiler et al. 2003):

$Q_{\mathcal{M}}=Q_{\mathcal{N}}(4 \pi / 3)\left[\int \rho_{\mathrm{d}}(a) a^{3} f(a) \mathrm{d} a\right]$.

Here, $Q_{\mathcal{N}}$ is the dust number rate; $\rho_{\mathrm{d}}(a)$ is the density of a grain, which depends on a grain radius $a ; f(a)$ describes the differential particle size distribution. The limits of integration are the minimal and maximum grain radii. In the expression

$$
Q_{\mathcal{N}}=\frac{A f \rho}{2 \pi^{2} p(\lambda) \Phi(\alpha) \int\left(f(a) a^{2} / v(a)\right) \mathrm{d} a}
$$

$p(\lambda)$ is a mean geometric albedo for the given wavelength referring to the mix of grain sizes; $\Phi(\alpha)$ is the phase function depending on a phase angle $\alpha$; and $v(a)$ is the ejection (terminal) velocity of a particle of a radius $a$. In order to obtain a quantitative estimate of the dust mass production, several realistic assumptions of the parameters used should be made:

Grain radius limits: comae of distant active objects are likely populated by large particles according to the numerical modeling of their dust environments (Fulle 1994; Korsun 2005; Mazzotta Epifani et al. 2009; Korsun et al. 2010). Therefore, we put the lower and upper limits on dust grain radii at 5 and $1000 \mu \mathrm{m}$, respectively.

Differential particle size distribution: we adopted the particle size distribution function in the form of $f(a) \sim\left(1-a_{0} / a\right)^{M} \times$ $\left(a_{0} / a\right)^{N}$, where $a_{0}$ is the minimum grain radius, $N$ is a slope of the function at large values of $a$, and the parameter $M$ locates the maximum of the distribution (Hanner 1983). We fixed $M$ and $N$ at 27 and 4 , respectively, which corresponds to the peak radius of about $40 \mu \mathrm{m}$.

Size dependent density: the expression for dust density was taken from Newburn \& Spinrad (1985): $\rho_{\mathrm{d}}(a)=\rho_{0}-\rho_{1}(a /(a+$ $\left.a_{2}\right)$ ) with $\rho_{0}=3000 \mathrm{~kg} \mathrm{~m}^{-3}, \rho_{1}=2200 \mathrm{~kg} \mathrm{~m}^{-3}$, and $a_{2}=2 \mu \mathrm{m}$. This gives to the smallest particles a density of $1.3 \mathrm{~g} \mathrm{~cm}^{-3}$ and the largest particles, which are thought to be more fluffy and porous, a density of $0.8 \mathrm{~g} \mathrm{~cm}^{-3}$. 
Table 5. Dust mass production rate of S3.

\begin{tabular}{lccc}
\hline \hline$r_{\mathrm{h}}(\mathrm{au})$ & $v(r) \mathrm{m} \mathrm{s}^{-1}$ & $\begin{array}{c}Q_{\mathcal{M}} \mathrm{kg} \mathrm{s}^{-1} \\
p(\lambda)=0.25\end{array}$ & $\begin{array}{c}Q_{\mathcal{M}} \mathrm{kg} \mathrm{s}^{-1} \\
p(\lambda)=0.1\end{array}$ \\
\hline 13.85 & $1-14$ & 3 & 9 \\
10.25 & $1-16$ & 4 & 11 \\
10.03 & $1-16$ & 6 & 15 \\
7.15 & $1-20$ & 10 & 26 \\
5.56 & $2-22$ & 21 & 52 \\
6.12 & $2-22$ & 33 & 82 \\
6.12 & $2-22$ & 31 & 78 \\
7.19 & $1-20$ & 25 & 62 \\
\hline
\end{tabular}

Dust outflow velocity: $v(r)$ was calculated with the equation taken from Sekanina et al. (1992). The parameters of the equation will be discussed below in Sect. 4.4. The calculated outflow velocities between 1 and $22 \mathrm{~m} \mathrm{~s}^{-1}$ are in agreement with the numerical modeling of dust environment of some other distant active targets at the same heliocentric distances (Fulle 1994; Mazzotta Epifani et al. 2009).

Grain albedo and phase function: although a value of 0.1 is often used in the literature we fixed the mean geometric albedo $p(\lambda)$ in the Eq. (3) at 0.25. Gehrz and Ney (1992) presented the observations of some comets and showed that their albedo lies in the range of $0.1-0.3$ on average, but the grains of the individual comets can display a high degree of variability.

Since the observations were made under low phase angles $\alpha$, the phase function was adopted in the form of $10^{-0.4 \beta \alpha}$. The phase coefficient $\beta$ was fixed at the mean value of 0.02 in accordance with the results presented by Meech \& Jewitt (1987).

The calculated values of dust production rate for the observed heliocentric distances are presented in Table 5. The table lists the dust production for two different albedo values, 0.1 and 0.25 , to facilitate comparison with results published by other authors, as well as the outflow velocities for the largest and smallest particles.

Shi et al. (2014) estimated the S3 dust production of about $360 \mathrm{~kg} \mathrm{~s}^{-1}$ at a distance of 5.86 au. This is about six times higher than that presented here for similar heliocentric distances. We managed to reproduce the same high dust production using the same set of the parameters Shi et al. (2014) used: grain albedo of 0.04 , density of grain material of $0.49 \mathrm{~g} \mathrm{~cm}^{-3}$, and grain outflow velocities of about $52 \mathrm{~m} \mathrm{~s}^{-1}$. This modeling demonstrates the influence of the input set of parameters on the calculated dust production rate. The grain albedo and outflow velocities are especially critical and can considerably change the calculated values. The dust production rate $\mathrm{S} 3$ calculated here is similar to those obtained for some other distant objects moving at the same heliocentric distances (Mazzotta Epifani et al. 2014; Mazzotta Epifani et al. 2009; Fulle 1994). For example, the dust production rate of comet C/2003 O1 (LINEAR) was estimated between 30 and $4 \mathrm{~kg} \mathrm{~s}^{-1}$ in the range of heliocentric distances of 7.01 au (pre-perihelion)-7.39 au (post-perihelion), the dust production rate of (2060) Chiron was estimated to be about $20 \mathrm{~kg} \mathrm{~s}^{-1}$ at a heliocentric distance of $11.2 \mathrm{au}$. Note that the dust production rate of S3 between 10.03 and 13.85 au is comparable to that of comet C/2002 VQ94 between 9.86 and 12.59 au $\left(3-6 \mathrm{~kg} \mathrm{~s}^{-1}\right)$ although S3 was on the inbound leg of its orbit and VQ94 moved from the Sun gradually fading (Korsun et al. 2014).

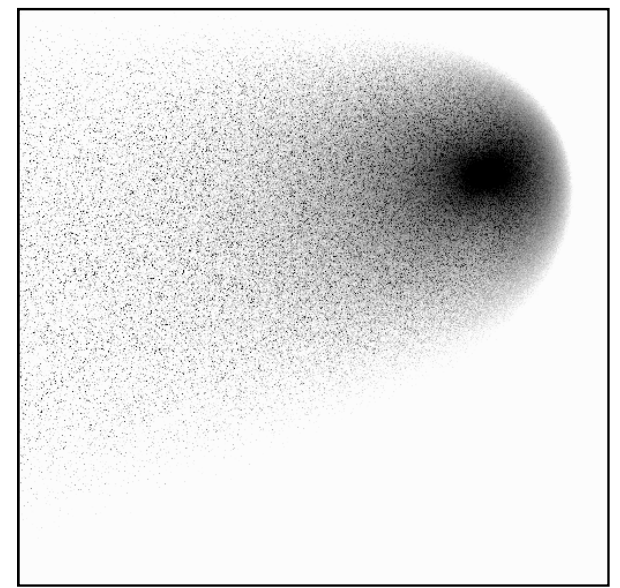

Fig. 7. Appearance of a tail modeled assuming uncollimated source.

\subsection{Dust environment modeling}

The comet showed prominent dust coma and tail during the 2013 observing session (see Fig. 1). A Monte Carlo model was used to fit the observed dust environment (Korsun et al. 2010). Greenberg's model was adopted to describe the chemical composition of the cometary dust particles (Greenberg \& Hage 1990). The formation of the comet tail by "dirty" ice particles with variable masses due to sublimation in the solar radiation field was considered. The time of S3 discovery was adopted as the starting time in our model runs. It means that the oldest particles were traced within 2006-2013 time interval. About $5 \times 10^{9}$ test particles were emitted along the considered arc of the orbit to gain sufficient statistics. The reason is the loss of a significant amount of test particles due to their escape from the examined frame and/or total sublimation. The dust model parameters, which were used to fit the observed dust structure of comet C/2003 WT42 (LINEAR) (Korsun et al. 2010) and expected to be effective in the S3 case, were adopted as a first approximation in the model runs. A trial and error approach was used to arrive at a likely solution with respect to our data. We obtained the result depicted in Fig. 7.

The result looks like the observed appearance of S3 in 2012. A large collection of appropriate images of S3 confirms this and is available on the Internet ${ }^{3}$. An additional narrow tail-like feature was apparent in 2013. We took into consideration this observational element for improving our model attempts. We managed to solve the problem of this narrow tail-like feature by including an additional source starting its activity at the beginning of 2013.

Our best fit is shown in Fig. 8. It was obtained using the following possible scenario for formation of the dust environment of the comet: (i) a rotating nucleus that had a rotation period of $15 \mathrm{~h}$; (ii) most of the surface of the nucleus was active; (iii) the side facing the Sun was only twice as active as the night side; (iv) the sizes $a$ of the tracing particles ranged within $5-1000 \mu \mathrm{m}$ with an $a^{-3.5}$ exponential dust size distribution; and (v) escaping velocities were controlled by a relation proposed by Sekanina et al. (1992):

$v_{\mathrm{d}}=\left(A_{0}+B_{0} \sqrt{a}\right)^{-1} r^{-0.5}$

3 See: http://www . aerith.net/comet/catalog/2006S3/ pictures.html, http://www.astrosurf.com/cometas-obs/ and kometen.fg-vds.de/pix/2006S3_e.htm 
Table 6. Model parameters used for our dust environment modeling.

\begin{tabular}{lcc}
\hline \hline Parameter & Source 1 & Source 2 \\
\hline Start activity & September 2006 & January 2013 \\
Inclination of the spin axis & $5^{\circ} \longleftrightarrow 0^{\circ}$ & $5^{\circ} \longleftrightarrow 0^{\circ}$ \\
Source latitude & $0^{\circ} \longleftrightarrow-5^{\circ}$ & $0^{\circ} \longleftrightarrow-5^{\circ}$ \\
Collimation & Uncollimated & Cone, half angle $=30^{\circ}$ \\
Dust production & \\
"Day/night" activity & $90 \%$ & $10 \%$ \\
Solid sizes & 2.0 & 2.0 \\
Exponential power index, $a^{x}, x=$ & $5-1000 \mu \mathrm{m}$ & $5-1000 \mu \mathrm{m}$ \\
$B_{0}\left(\right.$ Eq. $(4), A_{0}=0$ & -3.5 & -3.5 \\
Velocities $(a=5 \mu \mathrm{m}, r=5.9 \mathrm{au})$ & 0.0076 & 0.0212 \\
\hline
\end{tabular}

Notes. ${ }^{(a)}$ During 2013.

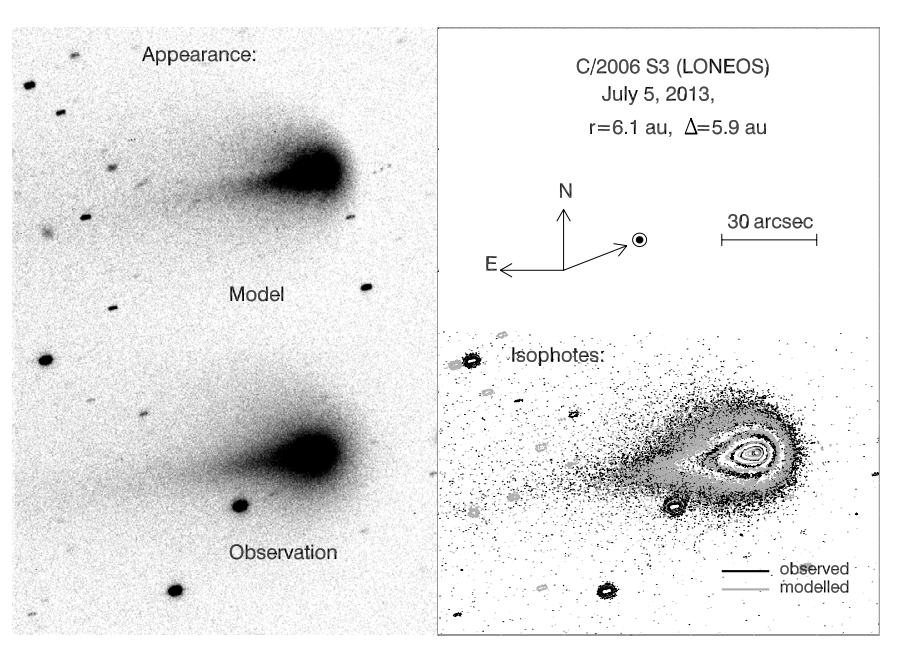

Fig. 8. Dust environment of S3 observed on July 5, 2013 (NOT data). The modeled brightness distribution is overlaid on the observed images and shifted for clarity (left panel). The right panel illustrates the contour plots of observed and simulated brightness with dark and gray lines, respectively. The scale bar of $30^{\prime \prime}$, directions to north and east, and the Sun are marked.

where $A_{0}$ and $B_{0}$ are model parameters. Here we used $v_{\mathrm{d}}=$ $(0.0076 \sqrt{a})^{-1} r^{-0.5}$. Heliocentric dependence of the dust production rate was adopted as $r^{-2}$.

An additional zone producing somewhat collimated outflow was activated at the beginning of 2013. Solid particles were emitted inside a cone with a half-width of $30^{\circ}$. The dust sizes, dust size distribution, heliocentric dependence, and (day side)/(night side) ratio of the dust production rate were the same as for the main source, while escaping velocities of the dust particles were rather low and controlled by $v_{\mathrm{d}}=(0.0212 \sqrt{a})^{-1} r^{-0.5}$. The area produced about $10 \%$ of a fresh supply of dust into the surroundings of the nucleus.

There still remains uncertainty as the orientation of the comet spin axis and the cometocentric latitude of the active zone. Close results of the simulation are achieved if the spin axis of the nucleus is normal to the plane of the orbit of the comet, and the active zone is located at a cometocentric latitude of $-5^{\circ}$, or the spin axis of the nucleus is deviated from the normal by $5^{\circ}$, and the active zone is located on the equator of the comet's nucleus. An intermediate combination of these parameters provided acceptable results.

The model parameters used in the model runs are presented in Table 6.

\section{Conclusions}

After eight years of monitoring the cometary activity of S3, it appears that this distant comet has been a very active object. The main results provided by our observations can be summarized as follows:

(i) The cometary activity level, measured with the Af $\rho$ parameter, appears to be more important after perihelion than before. At its maximum Af $\rho \sim 4000 \mathrm{~cm}$ in the $V$ band while at its minimum (at 13.67 au from the Sun, before perihelion) Af $\rho \sim 700 \mathrm{~cm}$.

(ii) When a mean geometric albedo $p(\lambda)$ is taken equal to 0.1 and grain outflow velocities in the range $2-22 \mathrm{~m} \mathrm{~s}^{-1}$, the corresponding dust production rate can be computed to reach $82 \mathrm{~kg} \mathrm{~s}^{-1}$.

(iii) No emission lines could be detected with our spectroscopic observations, both for heliocentric distances equal to 13.67 au and 9.97 au.

(iv) Our dust environment modeling is in agreement with a collimated source of dust that appeared in 2013.

Further observations of S3 in the coming years should also be useful to detect the changes of behavior in its cometary activity pre- and post-perihelion. Because of the high Af $\rho$ values computed for large heliocentric distances pre-perihelion, we can expect that $\mathrm{S} 3$ is among the more active objects observed in the solar system.

Acknowledgements. The results are based on the observations made with the $6 \mathrm{~m}$ telescope of SAO RAN, the $2 \mathrm{~m}$ telescope of Peak Terskol Observatory, the Nordic Optical Telescope (NOT), and the Liverpool Telescope (LT). The NOT is operated on the island of La Palma jointly by Denmark, Finland, Iceland, Norway, and Sweden, in the Spanish Observatorio del Roque de los Muchachos of the Instituto de Astrofisica de Canarias. The LT is operated on the island of La Palma by Liverpool John Moores University in the Spanish Observatorio del Roque de los Muchachos of the Instituto de Astrofisica de Canarias with financial support from the UK Science and Technology Facilities Council. The research leading to these results has thus received funding from the European Community's Seventh Framework Programme (FP7/ 2007-2013 and FP7/2013-2016) under Grant Agreements No. RG226604 and 312430 (OPTICON). This work was also supported by State Agency for Science, Innovation and Informatization (M/6 2013 agreement, Ukraine) and Campus France in the frame of a French-Ukrainian project "PHC Dnipro"

\section{References}

Afanasiev, V. L., \& Moiseev, A. V. 2005, Astron. Lett., 31, 194

A'Hearn, M. F., Schleicher, D. G., Millis, R. L., Feldman, P. D., \& Thompson, D. T. 1984, AJ, 89, 579

Bessell, M. S. 1999, PASP, 111, 1426 
Farnham, T. L., Schleicher, D. G., \& A'Hearn, M. F. 2000, Icarus, 147, 180 Filippenko, A. V. 1982, PASP, 94, 715

Fink, U., \& Rubin, M. 2012, Icarus, 221, 721

Fulle, M. 1994, A\&A, 282, 980

Greenberg, J. M., \& Hage, J. I. 1990, ApJ, 361, 260

Hanner, M. S. 1983, in Cometary exploration II., ed. T. I. Gombosi (CRIP, Budapest), 1

Haser, L. 1957, Bull. Soc. Roy. Sci. Liege, 43, 740

Kartasheva, T. A., \& Chunakova, N. 1978, Izvestija SAO (Rus.), 10, 44

Korsun, P. P. 2005, Kinematika i Fizika Nebesnykh Tel Suppl., 5, 465

Korsun, P. P., \& Chörny, G. F. 2003, A\&A, 410, 1029

Korsun, P. P., Kulyk, I. V., Ivanova, O. V., et al. 2010, Icarus, 210, 916

Korsun, P. P., Rousselot, P., Kulyk, I. V., Afanasiev, V. L., \& Ivanova, O. V. 2014, Icarus, 232, 88

Kulyk, I., Jockers, K., Credner, T., \& Bonev, T. 2004, Kinematika i Fizika Nebesnykh Tel, 20, 372

Landolt, A. U. 1992, AJ, 104, 340

Langland-Shula, L. E., \& Smith, G. H. 2011, Icarus, 213, 280

Levison, H. F. 1996, in Completing the Inventory of the Solar System, eds. T. Rettig, \& J. M. Hahn, ASP Conf. Ser., 107, 173

Marsden, B. G., \& Roemer, E. 1982, in Comet Discoveries, Statistics, and Observational Selection, ed. L. L. Wilkening, IAU Colloq., 61707
Mazzotta Epifani, E., Palumbo, P., Capria, M. T., et al. 2009, A\&A, 502, 355

Mazzotta Epifani, E., Perna, D., Di Fabrizio, L., et al. 2014, A\&A, 561, A6

Meech, K. J., \& Jewitt, D. C. 1987, A\&A, 187, 585

Meech, K. J., \& Svoren, J. 2004, Using cometary activity to trace the physical and chemical evolution of cometary nuclei (Tucson: University of Arizona Press), ed. G. W. Kronk, 317

Meech, K. J., Pittichová, J., Bar-Nun, A., et al. 2009, Icarus, 201, 719

Neckel, H., \& Labs, D. 1984, Sol. Phys., 90, 205

Newburn, R. L., \& Spinrad, H. 1985, AJ, 90, 2591

Newburn, R. L., Bell, J. F., \& McCord, T. B. 1981, AJ, 86, 469

Oke, J. B. 1990, AJ, 99, 1621

Prialnik, D. 1992, ApJ, 388, 196

Rousselot, P. 2008, A\&A, 480, 543

Sekanina, Z., Larson, S. M., Hainaut, O., Smette, A., \& West, R. M. 1992, A\&A, 263, 367

Shi, J. C., Ma, Y. H., \& Zheng, J. Q. 2014, MNRAS, 441, 739

Skiff, B., Christensen, E. J., Gibbs, A. R., et al. 2006, IAU Circ., 8752, 1

Tozzi, G. P., Boehnhardt, H., \& Lo Curto, G. 2003, A\&A, 398, L41

Weiler, M., Rauer, H., Knollenberg, J., Jorda, L., \& Helbert, J. 2003, A\&A, 403, 313 